\title{
Causal Co-personality: In defence of the psychological continuity theory
}

\author{
Simon Beck \\ School of Philosophy and Ethics \\ University of KwaZulu-Natal \\ Private Bag X01 \\ Scottsville, 3209 \\ South Africa \\ Email: beck@ukzn.ac.za
}

\begin{abstract}
The view that an account of personal identity can be provided in terms of psychological continuity has come under fire from an interesting new angle in recent years. Critics from a variety of rival positions have argued that it cannot adequately explain what makes psychological states co-personal (i.e. the states of a single person). The suggestion is that there will inevitably be examples of states that it ascribes wrongly using only the causal connections available to it. In this paper, I describe three distinct attacks on the psychological continuity theory along these lines. While I acknowledge that a number of interesting issues arise, I argue that the theory can withstand all three attacks.
\end{abstract}

\section{Introduction}

One common theme in criticisms of the widely-held psychological continuity theory of personal identity (hereafter 'PCT') is that it fails to provide an adequate account of co-personality, that is, of what makes two psychological states both states of one person. At least, that is the generalized claim that is made. When it comes to the detail, the argument is usually that the psychological continuity theory gets (some) ascriptions wrong, either ascribing certain states to someone when they are not states of that person, or not ascribing certain states that are theirs to them. This does indeed sound like a serious problem for this theory of personal identity. Some critics take it to be a reason to reject the theory outright, others argue that any remedy involves costs that may well be too high for the psychological theorist to accept. Either way, since similar criticisms arise from a variety of sources, it seems the issue deserves some attention. I will characterize the PCT, then describe three distinct attacks on it. While a number of interesting issues arise, I will argue that none of them succeeds in dislodging the PCT from its position.

The classic statement of the PCT was Derek Parfit's:

Psychological connectedness is the holding of direct psychological connections. Psychological continuity is the holding of overlapping chains of strong connectedness...For $X$ and $Y$ to be the same person, there must be over every 
day enough direct psychological connections.

(Parfit 1984: 206)

Parfit acknowledges that identity requires that psychological continuity be a unique relation - that is, only $Y$ must be continuous with $X$. He adds the important rider, however, that identity is not what matters - what matters is psychological continuity and connectedness; when we have that in a 1:1 relation, we can call it identity.

The connections that play the fundamental role in all of this are the connections of commonsense psychology - connections between experiences and later memories of those experiences, continuing dispositional states like belief and desire, the formation of an intention, the continued holding of that intention and its later execution, and so on. While Parfit's account focuses on conscious mental connections, there is no reason why unconscious connections should not also feature - some of the desires and beliefs connecting an earlier self to a later one may well be unconscious ones.

\section{Three attacks}

Why might such a theory fail as an account of co-personality? The point is made in a number of different ways. Geoffrey Madell confronts the PCT with this version:

the following two thought-experiments are equally intelligible: (a) that I might not have existed, but someone having exactly the life that I have had might have existed instead; (b) I might have had a totally different life, even to the extent of being born centuries earlier.

(Madell 1981: 79)

His point is that certain psychological states are just mine - they are not mine in virtue of being connected to other states or events, and their connections to other states cannot explain why it is that they are mine. Paul Ricoeur makes a similar claim: "the factual character" of certain psychological and physical events involves a "phenomenon of mineness" (Ricoeur 1992:132). This phenomenon of mineness cannot be reduced to impersonal causal connections between psychological states, and it will inevitably be lost in the attempt. As the charge suggests, the solution would have to be to give up the PCT in favour of a non-reductionist account of co-personality.

A second version of the charge (with echoes of the first) comes from Marya Schechtman. She argues that, just as Locke's original account of personal identity in terms of 'sameness of consciousness' failed to accurately ascribe states to people, so its successor - the PCT - fails. Locke's account would have it that (somehow) remembering someone else's experience makes you that person, and not being able to remember an experience makes it not yours. The PCT has gone some way to avoiding these implausible consequences, but (in Schechtman's view) not nearly far enough. It extends Locke's criterion in requiring 'enough' connectedness, rather than just one memory, and in including other psychological connections alongside memory, but this is not the way to solve the problem. The demand for 'enough' connections makes co-personality an arbitrary matter - whether or not these are your states can depend on the stipulation of what counts as 'enough'. Locke's requirement of direct (memory) access to an earlier experience at least explained why it was yours - why you were 'responsible to' it (Schechtman 2005: 16); removing the requirement of direct access while including psychological states other than memory loses that explanation. She sees no way in which the PCT can remedy this using only the causal connections that feature in it. Her solution is to reject the PCT and go narrative - for experiences, states 
and actions to be yours they must fit meaningfully into your self-conception. That meaningful fit explains why they are yours.

The final version that I will consider comes in the form of a counter-example from Nicholas Agar, ${ }^{1}$ the case of 'witnessed pain' (Agar 2003: 59-60). In the normal case of someone feeling pain, they believe they are in pain - the two states are co-personal, and the PCT explains this in terms of the belief being caused by the pain. Agar envisages a case in which $\mathrm{P}$ is in pain and this causes $\mathrm{Q}$ to believe that there is pain. The causal connection obtains, but that does not make the states co-personal: the belief in question belongs to $\mathrm{Q}$, not $\mathrm{P}$. The obvious response is for the PCT to require that more than just this one connection needs to be present: for the belief that there is pain and the pain to be co-personal, the belief must cause (or be causally related to) a desire to alleviate the pain. But this attempt at a remedy is the start of a slippery slope, according to Agar. It is easy to change the counter-example such that the extension does not help: Q happens to be a very sympathetic person, and the belief that there is pain causes Q to want to alleviate the pain. And if the proponent of the PCT requires a specification of content? That is, for the states to be co-personal, the belief caused must be more than just the belief that there is pain - it must be that the pain is mine. Agar replies:

It is possible that a person possessed of Jesus-level empathy may sincerely think 'that pain is mine' and have the desire to alleviate the pain without feeling the pain and therefore without the pain being copersonal with the belief. (Agar 2003: 60)

Agar does not reject the PCT as Madell and Schechtman do. He argues that it can be saved, but at some cost. To save it, what needs to be provided is a way of ruling out cases (like the case of witnessed pain) that are merely 'superficially causally isomorphic' (2003: 60) to the co-personal case. Agar suggests the following as a necessary condition for co-personality.

A necessary condition for two states to belong to the same person is that it is possible to trace a chain of actual and potential causes between them that includes no non-psychological intermediate.

(Agar 2003: 62)

In the genuinely co-personal case of pain and belief, there are no non-psychological intermediates. However, in the case of witnessed pain there are some. Q's belief that there is pain results from Q's observation of P's behaviour, such as the sound of P's groans. The sound waves that carry the groans to $\mathrm{Q}$ are superficially like the brain activity that links Q's pain to Q's belief, but apart from that single causal link their activity is quite different. The functional architecture of the brain is such that it can support psychological patterns - brain states are disposed to interact in the way in which psychological states interact, and thus brains realize psychology. ${ }^{2}$ Sound waves are not disposed to interact in that sort of way at all, and thus they do not realize psychologi-

1 It is worth noting that Agar does not actually present the thought-experiment so much as suggest that there might be such a thought-experiment. Perhaps this is because of his skepticism about their usefulness that I will refer to later. It remains true that his case for his necessary condition depends crucially on this thought-experiment as I outline.

2 Agar's remedy is rooted in a commonsense functionalist account of psychological states as the wording here suggests. I have taken his criticism of the PCT out of that context; I am not apologizing since I think that is important in grasping the structure of his case against the PCT. 
cal states. That means there is a psychological gap in the case of witnessed pain that does not occur in the genuinely co-personal case and the counter-example can be ruled out.

Agar is quick to point out that holders of the PCT might not find this remedy a welcome one, however. The reason is that as well as excluding the case of witnessed pain as a case of co-personality, the proposed condition also rules out brain-state transfer (BST) devices and teletransporters as means of maintaining co-personal links between states. Just as the sound waves in the current example represent a psychological gap, so will the workings of the teletransporter or the BST device. Thought-experiments involving devices like these play a prominent role in the arguments of proponents of the PCT: arguments that made the PCT an attractive theory in the first place. To accept Agar's remedy means giving them up. On his account, even though it is a psychology-based account, our survival will depend on the continued existence of our brains: 'Functionalism about mental states may abstract away from information about the physical nature of the realizers of mental states, but we should not extend such reasoning to the realizer of my identity' (2003: 68).

Which path is then the one to take? Do these arguments show the PCT to be inadequate, and if so, which offers the best remedy? I will argue that ultimately we are not provided with anything like enough reason to abandon the PCT as it stands.

\section{The non-reductionist attack considered}

Consider first the case for giving it up in favour of a non-reductionist view. The non-reductionist asserts that states either are mine or are not mine and that this is not in virtue of any other facts being the case. Madell's two thought-experiments are presented as evidence: if we accept that my mental states could have been totally different, ${ }^{3}$ then there is no grounding for the ones that happen to be mine being mine. But that does not strike me as being relevant evidence, at least it is not evidence relevant to the issue at hand. That my life could have been utterly different or that someone else could have lived a life exactly like mine tells us (if anything) something about our identity across worlds: it could be that $\mathrm{X}$ in world $u$ is me even though $\mathrm{X}$ and I have nothing else in common than that. But it tells me nothing about what is or is not required for my identity across time. ${ }^{4}$ The utterly different life that I lead in $u$ needs the events and states that make it up to be connected to each other in complex ways, otherwise it is not the life of a person at all. That is the issue we are facing here - co-personality within a world - and which is simply not addressed by Madell's conjectures.

It is true that we have a strong sense of experiences being ours and a sense that certain future ones will be ours in the same way. It may also be true that we don't apply criteria when thinking of our own future experiences - that is, we don't think of them as experiences connected to our current ones in any specific way. But that does not mean that this sense of 'mineness' is all there is to being a continuing self: having a sense of self is necessary to being a person, but it is not what makes you the same person over time. If later experiences are not causally connected to earlier ones in the right sort of way, then no sense of them being yours or not will make them yours or not. People suffering from 'thought insertion' experience certain thoughts as being

3 As an anonymous referee for this journal commented, this is a point that many might not accept, and that Madell is guilty of begging the question by assuming what we wants to show. I will let Madell have the point for argument's sake.

4 As I argued in Beck (1999). 
those of somebody else, but that neither makes them the thoughts of that other person, nor does it make them not the thoughts of the sufferer.

Madell has another charge against the PCT that is not as obviously guilty of any of the confusions I am suggesting here. This is the claim that the particular sorts of connection between experiences and other states that the PCT highlights are inadequate for the task at hand. What psychological connections cannot do, he suggests, is to explain our concern about future pain. Attempting to explain this concern in those terms

... makes it look as if what we are saying is that the real object of our concern is that the person who will be in pain will have certain memories and psychological traits; and it seems absolutely clear that this is not what one is afraid of at all. What one is afraid of is just that the person who will be in pain is oneself.

(Madell 1981: 15)

This seems to be another way of putting the claim that it is not the psychological connections between states that makes those states mine. It is rational to fear that I will feel pain in the future, but not rational to fear that the person with such-and-such mental states will feel pain in the future, and thus the PCT has not captured what it is about those states that makes them mine.

Although this appears to be closer to the mark, it is not clear that it does any more damage than the first argument. Apart from the obvious response that the failure to substitute in an intensional context does not mean that the expressions in question are not identical ones, considerations like those raised earlier are still pertinent here. What makes it rational for me to fear future pain is once again my sense of self: I have a sense that it will be my pain. To be a person, you need to have a sense that experiences were yours in the past and will be yours in the future. The PCT is perfectly capable of including thoughts like these; nothing in it is at odds with this requirement. That we don't overtly link particular connections between our current states and later states when we think of future thoughts as being ours does not mean that such connections are not crucial in making those thoughts ours: it is simply a matter of how the future thoughts are presented.

\section{The attack from self-understanding}

As a result, the reasons non-reductionists offer as counting against the PCT are not sufficient to require its rejection. There are other reasons for not wanting to adopt a non-reductionist solution as well, one of which is relevant to a criticism of Schechtman's, to which I now turn. Schechtman points to the problem Locke faced in that his account wrongly implies that if I have a memory of someone else's experience, then I am identical to that person. She rejects the amendment that the PCT makes in requiring that there be 'enough' connections between earlier and later stages for them to be co-personal, on the grounds that any stipulation of what counts as enough will be an arbitrary one (Schechtman 2005: 12). Presumably the idea behind her charge is that whether or not states are co-personal cannot be an arbitrary matter. On this point, she is in agreement with the non-reductionist: Madell would agree that such a thing cannot be a matter of convention. But this is a point on which proponents of the PCT have engaged their opponents. A central thrust of Parfit's argument for his view is an argument designed to show precisely that identity (or co-personality) is not necessarily determinate or non-arbitrary - that argument being his 'Combined Spectrum' thought-experiment (Parfit 1984: 236-243; 1995: 21). Whether or not you find 
that argument convincing, its conclusion seems to find fairly obvious support elsewhere. Sufferers of senile dementia or diseases like Huntington's corea show a gradual psychological disintegration. Before the disease sets in we have a clear case of a person; at the end we have an organism that is no longer a person (on any of the accounts we are considering). That means somewhere in the process of disintegration, states ceased to be co-personal. To specify a point would be arbitrary, and that is precisely the message of the PCT's reductionism. But doesn't that mean that Schechtman is correct? By demanding 'enough' connections, she is suggesting that the PCT is implying that up until that degree of connectedness states are not co-personal, but once it is reached they are. But that is not the implication at all. Parfit writes:

we can claim there is enough connectedness if the number of direct connections, over any day, is at least half the number that hold, over every day, in the lives of nearly every actual person.

(Parfit 1984: 206)

Schechtman seems to reading this as a specification of a necessary condition on co-personality, but it is not that. Parfit makes it clear that his view is that there can be cases where the difference between the presence and absence of co-personality is an empty question. What he is offering here is an example of a case which is clearly not one of those - he is not specifying a threshold. Schechtman's criticism is thus misleading, and simply does not count against the PCT.

Her second point outlined above is more noteworthy. This is the argument that in giving up Locke's requirement of direct memory connections for two stages to be co-personal, the PCT avoids one problem but faces an equally grave one. The PCT accepts that an experience can be yours even though you have forgotten it, whereas Locke is obliged to (implausibly) deny this. As long as there are overlapping connections forming a link back to the experience, the PCT marks it as yours. What this loses, in Schechtman's view, is any way of explaining what makes them ours. Of course, the PCT is offering an explanation of what unifies a person's experiences they are all part of one causally connected psychological continuum - but she wants to argue that is not enough: this does not explain all that it should. Indicating what is omitted, she writes

Experiences of which we are directly conscious ... are also, at least according to Locke, tied to responsibility in this way, because we can know them to be our actions or experiences, we have a responsibility to and for them that we could not otherwise have.

(Schechtman 2005: 15-16)

The PCT counts actions and experiences of which we (now) have no consciousness as ours and thus for which we are responsible. But what sense is there in holding someone responsible for something of which they are not even aware?

None of this strikes me as being particularly clear or justified. On the clarity side, I'm not sure what sense to make of the notion of someone's 'responsibility to' their experiences. My current psychological states are responsive to my earlier states in that their existence and their features are the result of earlier states and experiences: those earlier episodes explain why I am like I am today. But this presents no problem for the PCT - this is precisely what the theory suggests and it is unproblematic that I cannot remember (even important) formative experiences - that does not make them not 
mine, and their role in bringing about and thus explaining later states seems to satisfactorily explain them as mine: they played no such role in the formation of anyone else's states. Schechtman's notion of 'responsibility to' seems to be pointing to something other than this notion of responsiveness, then.

Perhaps we can get a clue from the problem involved in holding someone responsible for actions of which they are not conscious. The difficulty here is that this problem is not all that much clearer. In the first place, the PCT may well be better off than Locke on the question of a link between responsibility and awareness. It is perfectly understandable for you to accept responsibility for things you cannot remember doing, such as things you only know about through stories in family lore of your long ago behavior (Goldie 2011). And you can be unaware of what you are doing and still be responsible for doing it - you ought to have been paying attention, and so on. Moreover, consciousness of an action being yours does not make you responsible for it. This leads to a second point. Proponents of the PCT are aware that aspects of their view imply that the link between personal identity (or co-personality) and responsibility is not the very strong one that many claim to see in commonsense morality - that you are responsible for all and only your actions (a responsibility that admits of no degrees). Parfit has argued eloquently that commonsense morality is much less strict than this view allows, and that degrees of responsibility are widely recognized and fit well with the reductionist metaphysics of the PCT. And there are other points that he might well have raised in questioning the supposed link and any consequent issues that the PCT is required to explain. For instance, responsibility is sensitive to many factors other than co-personality. Actions may be yours and yet your lack of autonomy means you are not responsible for them. Others can take responsibility for things that you did. A theory of co-personality may well have something to say about some cases of responsibility, but since co-personality is neither necessary nor sufficient for responsibility, it is unclear that it can be held to task for failing to explain responsibility.

As a final attempt to understand Schechtman's complaint, let us consider the solution she offers to the problem she sees. On her 'self-understanding' view, what makes experiences or psychological states yours is that they fit meaningfully into your life story. With Locke's requirement for direct awareness, we know these experiences to be ours. In her account, because these experiences fit meaningfully, we understand them to be ours. They are part of my sense of self. In 'Personality and persistence' (2004), she suggests that this involves empathizing with past attitudes as well as just remembering them as yours (2004: 94). We are on familiar territory here - this is the notion of a sense of self that I discussed in the context of non-reductionist views, now made more concrete as a narrative theory. But while spelling out the details of how our sense of self has or needs to have narrative features might provide us with a better account of our sense of self, it does not provide any better account of co-personality. I pointed to the reasons earlier, but it might be worthwhile spelling them out more clearly.

Having a sense of experiences as yours - as fitting in to your life story or self understanding - does not make them yours. Someone who has heard of an experience and then assimilated it amongst their own and now tells the story of that experience as theirs has done just what this description suggests - fitted someone else's experience into their life story. The experience might even become crucial to their being the sort of person they see themselves as. But it remains that the experience was not theirs. On the other side, that an experience does not fit into your sense of self does not mean it is 
not yours. This is so of suppressed experiences (which Schechtman does discuss) and even more graphically of experiences of sufferers of thought-insertion (which she does not). The case of thought-insertion is of the experience of other people's thoughts occurring in your mind: not just that they are influencing your thoughts, but that they are using your mind to think their thoughts. The sufferer is certain that these are not their own thoughts, and they do not fit into their own self-understanding in any way: that is precisely what they do not do (Mullins and Spence 2003). Thought- insertion is a pathology - a disease affecting self-understanding. And it is a graphic illustration of why the sense of self is not a guide to co-personality.

Schechtman's discussion of unconscious states is also illuminating here. She accepts that such states may well be central to the psychological life of a person even though that person may have no conscious access to them at all, as in the case of suppressed experiences. This leads to her view finally being expressed in the following terms.

Those memories or desires or motivations whose existence as part of the psychological economy must be postulated in order to make sense of a person's experience or the course of her life will be considered her experiences.

(Schechtman 2005, 20)

In this expression of the view, it is crucially important that the person's own self-understanding is no longer the final determinant of co-personality. If we need to ascribe suppressed desires, beliefs or experiences to someone in order to make sense of their lives, then those experiences are theirs even if they understand themselves very differently - whether they 'recognize this fact or not' (2005: 20). This is doubly interesting in the light of her attack on the PCT. First, it undermines her criticism that the PCT does not capture what makes experiences ours as her theory (and Locke's) can. What makes states ours for the PCT is that they have a place in the causal story that leads up to our current states - their part in that causal continuum. What makes unconscious states ours for Schechtman is that we need them to explain why the person behaves as they do. If the PCT is in trouble, the self-understanding view is in the same trouble: to all intents and purposes, their accounts of the co-personality of these important states are the same.

Second, as this point suggests, there are tensions in Schechtman's account that are coming to the fore - tensions between someone's own self-understanding and how others understand them. It is clear that the two need not co-incide; if the psychological evidence of self-enhancement biases as normal is anything to go by (Brown 1986), the two will often not co-incide. Schechtman's account needs an emphasis on our own self-understanding in places, yet has to deny the importance of that altogether in others. The PCT on the other hand, shows no such tension. It neatly includes suppressed states as co-personal with conscious states and has no difficulty with pathologies like thought-insertion. Schechtman implies that it cannot cope with abnormal psychology suggesting an unfortunate chauvinism; but it is not the PCT which is in trouble here at all.

\section{Agar's attack}

The picture presented so far has been of critics saying that the PCT does not offer enough to explain what makes states yours and my responses that the critics are asking too much - when more is added to what the PCT offers, the result is mistaken ascriptions of co-personality. Agar's criticism is different from that of the others, however. 
He has argued that the PCT includes too much - it implies that certain states which are not ours are ours. His solution is to limit the PCT in line with a particular aspect of commonsense functionalism regarding mental states. With this tightening of the necessary conditions on co-personality, the counter-example of witnessed pain can be excluded. As explained earlier, he foresees some costs here for the PCT alongside the gains. These are worth some attention, but here I want to focus on his remedy for the witnessed pain problem.

Agar's proposed necessary condition was the principle he calls ' $\mathrm{F}$ ':

(F) A necessary condition for two states to belong to the same person is that it is possible to trace a chain of actual and potential causes between them that includes no non-psychological intermediate.

(Agar 2003: 62)

He points out that there is no such appropriate chain between P's pain and Q's belief that there is pain, even though there is a causal chain between the two. That means his condition marks them as not co-personal, whereas the PCT marks them (or appropriately described states if the example needs to be more complex) as co-personal. So, Agar's criterion produces the right answer - but does it solve the problem in the right way? There are a number of ways in which it might fail - it might itself rule out actual cases of co-personality or there might be other ways of solving the problem that offer more intuitively plausible accounts of co-personality. This latter point may well be important. Agar offers little by way of arguing for the plausibility of his principle as a necessary condition on co-personality. He argues that it is consistent with commonsense functionalism, but otherwise its only serious motivation is that it gets the right answer to the witnessed pain case.

Is his condition too strong in the sense that it rules out cases of co-personality? Things get rather murky here. The obvious cases that holders of the PCT might point to will be counterfactual thought-experiments and Agar is willing to dig in his heels here and say that these do not count - our concept of a person is 'a patchwork of different theoretical considerations' and as such is 'an inappropriate tool to make the fine discriminations' such thought-experiments trade on (2003: 68). His own thought-experiment is apparently to be placed in a different category - of cases where states are 'patently not unified' (2003: 59). That his necessary condition works in such an obvious case where the unamended PCT fails justifies rejecting intuitive responses to more outlandish thought-experiments involving teletransporters and so on as false positives. This is too tricky an issue to resolve here: all I will do is raise an eyebrow and go on to investigate the other possible course of failure.

I will argue that there is an alternative and more intuitively plausible way of resolving the witnessed pain problem, and one that is implicit in Agar's own resolution.

Agar points to the role of the sound waves in the witnessed pain story as the problematic aspect. Q's belief that there is pain is caused by the sound waves that bring P's groans (caused in turn by P's pain) to Q. These sound waves do not realize psychological states. That means there is a psychological gap - a 'gap in thinking' - between the pain and the belief. It is this gap that means the states are not co-personal: 'failure to satisfy F means that states are not unified' (2003: 65). As I said, Agar does not do much to make his necessary condition intuitively plausible, and this is about the extent of what he offers. It is enough to lead me to my alternative resolution, however. 
Consider his explanation as to why the sound waves are not psychological intermediates. It is not because they are physical phenomena - other physical phenomena like brain states can be psychological intermediates. What they lack is provision of functional architecture - they do not have the necessary causal dispositions to realize psychological states. Following commonsense functionalism, psychological states are defined by their causal roles - they tend to be caused in typical ways, to interact typically with other psychological states and (with other such states) typically result in certain types of behavior. Psychological states are supervenient; the roles that define them are played by first-order states, states which must have isomorphic causal dispositions if they are to play the roles. The sound waves are only superficially isomorphic: they cause the belief in $\mathrm{Q}$, but fail to have any of the other complex dispositional relations to internal states in Q that would make them realizers of the role of a psychological state.

Agar is right that the sound waves don't realize psychological states. But is he right that it is failure to satisfy F that makes the pain and the belief not co-personal? For the witnessed pain case to count as an argument against the PCT, and as a result to give us a reason to believe that $\mathrm{F}$ is interesting as a necessary condition for co-personality, it must be the case that the PCT cannot explain away witnessed pain with its own resources and without Agar's help. Agar found fault with sound waves because they do not instantiate the required patterns, and that shows us the obvious way - it's not so much the instantiation of patterns that is missing, but the patterns that require instantiation in the first place. The commonsense pattern that initiates witnessed pain is that pain causes someone to believe that they are in pain (this points to an immediate oddity in the vague description that Agar offers - has anyone ever believed that 'there is pain'? What sort of belief is that?) To be a belief that I am in pain is (on the commonsense functionalist account to which Agar appeals and to which I have no objection) to be a state caused by pain and which interacts with a complex pattern of other states. Primary amongst these is a memory of what the pain felt like on its first occurrence and what it feels like now, which point in your body it affects, and many related experiences and memory experiences. These will include both 'field' and 'observer' perspectives - remembering how strong it was and remembering yourself as if from the outside reacting to the pain (see Sutton 2010 for how these are part of everyday familiar memory experiences). There will also have to be the desire to alleviate the pain that Agar mentions along the line as well as everything else that is part of how we experience and understand the causal role of pain.

Now consider Q's belief again. Is there any reason to think that Q's belief that 'there is pain' or that 'this pain is mine' is co-personal with P's pain on the PCT? The patterns that make up overlapping chains of psychological connections are the patterns of commonsense psychology that functionalists highlight - for instance, you can't intend to do something without choosing to do it and without having some second-(or third-) order states that prevent you from changing that plan too easily. They are complex patterns, and the PCT points to this complex interconnectedness in its picture of co-personality. As Schechtman realized with the PCT's move away from Locke's direct memory, isolated states simply don't feature in the continuum - there is nothing that commits the PCT to Q's (odd) beliefs being co-personal with P's pain. That is important, because it means that the failure of the sound waves to realize patterns in Agar's case is simply irrelevant. If we needed F to block the inference from PCT to co-per- 
sonality in the case things would be otherwise, but without this support for it we have no serious reason to see Agar's proposal as significant.

Agar, as we have seen, suggests that a counter-example can be set up that works even if we require more complex states on behalf of $\mathrm{Q}$, such as his suggestion of someone with Jesus-level empathy whose states still remain separate from those of the feeler of the pain. But his sketch is simply inadequate - if the details I am suggesting above are added in, and Q has all those states, then it becomes very hard to deny that $\mathrm{Q}$ felt the pain. The case would be peculiar, but a peculiar case of co-personality. At the very least, it is far from the case of patent non-unification that Agar's argument demands.

The PCT suggests that it is complex causal interaction with other states that makes psychological states yours. Agar's F condition suggests that it is a lack of non-psychological intermediates that makes them yours. Admittedly, this is slightly unfair since he is only offering a necessary condition, not a necessary and sufficient condition, but it does highlight that the PCT has an independent plausibility that Agar's F lacks. Agar might well insist that commonsense functionalism and its view on realization make $\mathrm{F}$ a necessary requirement on co-personality, but that is not clearly true. Whatever its policies on realization, folk psychology might well allow us to survive gaps, and it is the gap between pain and belief that seems to be doing the work in Agar's argument against PCT. Once again, justifying his condition will require more intuitive appeal than he is offering, and that might well involve appeal to thought-experiments like those he eschews.

My conclusion is that of the four alternatives considered, the PCT provides the most satisfactory account of co-personality. None of the criticisms we have seen require us to give it up in favour of another view. ${ }^{5}$

\section{References}

Agar, N. 2003. 'Functionalism and personal identity'. Nous 37(1), pp52-70.

Beck, S. 1999. 'Leibniz, Locke and I'. Cogito 13(3), pp181-187.

Brown, J. 1986. 'Evaluations of self and others: self-enhancement biases in social judgements'. Social Cognition, 4, pp353-76.

Goldie, P. 2011. 'Empathy with one's past'. Forthcoming in Southern Journal of Philosophy.

Madell, G. 1981. The identity of the self. Edinburgh: Edinburgh University Press.

Mullins, S. and Spence, S. 2003. "Re-examining thought insertion". British Journal of Psychiatry 182, 293-298.

Parfit, D. 1984. Reasons and Persons. Oxford: Clarendon Press.

Parfit, D. 1995. 'The Unimportance of Identity', in Harris, H. (ed.). Identity. Oxford: Clarendon Press.

Ricoeur, P. 1992. Oneself as Another, trans. Kathleen Blamey. Chicago: University of Chicago Press.

Schechtman, M. 2004. 'Personality and Persistence'. American Philosophical Quarterly 41(2), 88-105.

5 I am grateful to Jacek Brzozowski and an anonymous referee for comments on an earlier draft of this paper. 
Schechtman, M. 2005. 'Personal identity and the past'. Philosophy, Psychology and Psychiatry 12(1), pp9-22.

Sutton, J. 2010. 'Observer perspective and acentred memory: some puzzles about point of view in personal memory'. Philosophical Studies 148, pp27-37. 